\title{
Delineating antibody recognition against Zika virus during natural infection
}

Lei Yu, ${ }^{1}$ Ruoke Wang, ${ }^{2}$ Fei Gao, ${ }^{2}$ Min Li, ${ }^{3}$ Jianying Liu, ${ }^{4}$ Jian Wang, ${ }^{1}$ Wenxin Hong, ${ }^{1}$ Lingzhai Zhao, ${ }^{1}$ Yingfen Wen, ${ }^{1}$ Chibiao Yin, ${ }^{1}$ Hua Wang, ${ }^{2}$ Qi Zhang, ${ }^{2}$ Yangyang Li, ${ }^{2}$ Panpan Zhou, ${ }^{2}$ Rudian Zhang, ${ }^{4}$ Yang Liu, ${ }^{4}$ Xiaoping Tang, ${ }^{1}$ Yongjun Guan, ${ }^{1}$ Cheng-Feng Qin, ${ }^{5}$ Ling Chen, ${ }^{6,7}$ Xuanling Shi, ${ }^{2}$ Xia Jin, ${ }^{3}$ Gong Cheng, ${ }^{4}$ Fuchun Zhang, ${ }^{1}$ and Linqi Zhang ${ }^{2}$

'Guangzhou Eighth People's Hospital, Guangzhou Medical University, Guangzhou, China. ${ }^{2}$ Comprehensive AIDS Research Center, Collaborative Innovation Center for Diagnosis and Treatment of Infectious Diseases, Department of Basic Medical Sciences, School of Medicine, Tsinghua University, Beijing, China. ${ }^{3}$ Viral Disease and Vaccine Translational Research Unit, Institut Pasteur of Shanghai, Chinese Academy of Sciences, Shanghai, China. ${ }^{4}$ Department of Basic Medical Sciences, School of Medicine, Tsinghua University, Beijing, China. ${ }^{5}$ State Key Laboratory of Pathogen and Biosecurity, Beijing Institute of Microbiology and Epidemiology, Beijing, China. ${ }^{6}$ State Key Laboratory of Respiratory Disease, First Affiliated Hospital of Guangzhou Medical University, Guangzhou, China. ${ }^{7}$ Guangzhou Institute of Biomedicine and Health, Chinese Academy of Sciences, Guangzhou, China.

Zika virus (ZIKV) is an emerging mosquito-transmitted flavivirus that shares a considerable degree of homology with dengue virus (DENV). Here, we examined longitudinal antibody response against ZIKV during natural infection in 2 convalescent individuals. By decomposing the antibody recognition into DI/DII and DIII of the E glycoprotein, we showed their development in humans followed a spatiotemporal hierarchy. Plasma binding to DI/DII appeared to peak and wane during early infection with extensive cross-reactivity with DI/DII of DENV. Binding to DIII, however, peaked early but persisted months into the infection without detectable cross-reactivity with DIII of DENV. A clear trend of increase in DIII-specific neutralizing activity was observed over the course of infection. mAbs isolated during early infection are largely DI/DII specific, weakly neutralizing, and highly cross-reactive with DENV, while those from later infection are more diverse in recognition, potently neutralizing, and ZIKV specific. The most potent neutralizing mAb targeting the DIII provided $100 \%$ protection in mice from lethal ZIKV infection and could therefore serve as a promising candidate for antibody-based therapy and prevention. The dynamic features unveiled here will assist us to better understand the pathogenesis of ZIKV infection and inform rational design of vaccines.

Authorship note: L. Yu and R. Wang are co-first authors. F. Zhang and $\mathrm{L}$. Zhang are co-senior authors.

Conflict of interest: The authors have declared that no conflict of interest exists.

Submitted: January 24, 2017

Accepted: May 10, 2017

Published: June 15, 2017

\section{Reference information:}

JCI Insight. 2017;2(12):e93042.

https://doi.org/10.1172/jici.

insight.93042.

\section{Introduction}

Zika virus (ZIKV) is a mosquito-transmitted flavivirus that can cause microcephaly in fetuses and newborn infants and is associated with Guillian-Barre syndrome in adults (1-6). Since its first discovery in a sick rhesus macaque in the Zika forest in Uganda (7), ZIKV has been expanding and adapting to human populations across broader geographic frontiers. Most notable was the recent unprecedented outbreaks in the Americas, Caribbean, and Southeast Asia $(8,9)$ through the invasive mosquito species Aedes aegypti, although the underlying biological and ecological mechanisms remain unclear. The high prevalence of this mosquito species coupled with climate changes is likely to fuel global spread of the epidemic, raising the serious concerns about pandemic potential of ZIKV in the near future $(10,11)$.

Neutralizing antibodies are the major component of protective immunity against viral infection in humans. Polyclonal by nature, they exert their functions through complex mixture of mAbs to target the crucial epitopes on the viral envelop glycoprotein. Identifying the neutralizing mAbs and their recognized epitopes has therefore become the first crucial step for a better understanding of the protective antibody response and for the development of antibody-based therapy and vaccines $(12,13)$. In human infection with ZIKV, dengue virus (DENV), and other flaviviruses, the surface envelope glycoprotein (E) that mediates viral infection is the major target for neutralizing antibodies (14-20). On a matured virion, there are 180 monomers of $\mathrm{E}$ glycoprotein that are packed into 90 head-to-tail homodimers 
(21-27). The individual subunit of E glycoprotein consists of $3 \beta$-barrel domains (DI, DII, and DIII) and is interconnected through flexible hinges $(21,22,28,29)$. DIII is believed to contribute to the viral attachment, while DII triggers fusion between viral and target cell membrane. Analysis of mAbs from ZIKV-infected humans and mice indicated that recognition of different domains is associated with distinct neutralizing activity in vitro and protective capacity in animal models (17, 30-32). In general, mAbs against DIII are type specific, some potent-neutralizing and protective, whereas those against DI/II are cross-reactive and poorly neutralizing, and they markedly enhance infection in vivo $(17,31)$. ZIKV infection also elicits antibodies that recognize complex quaternary epitopes composed of more than one domain or E glycoprotein as in other flavivirus infection (16, 19, 29, 31-34). However, development of these neutralizing antibodies against ZIKV and their cross-reactivity with DENV during natural infection remain largely unknown.

Here, we examine the kinetics of antibody responses against ZIKV during natural infection in 2 convalescent individuals. By sequential evaluation of binding specificity and neutralizing activity of polyclonal plasma and isolated mAbs from B cells, we have revealed complex and dynamic features of antibody response during development. In general, plasma binding to DI/II appeared to peak and wane during early weeks of infection and to extensively cross-react with DI/DII of DENV. In contrast, those against DIII peaked but persisted into an extended period without any detectable cross-reactivity with DIII of DENV. A clear trend of increase in DIII-specific neutralizing activity was observed over the course of infection, although a notable proportion of neutralizing activities were not accounted for, presumably due to those antibodies recognizing more complex quaternary epitopes composed of more than one domain or E glycoprotein. Furthermore, mAbs isolated during early infection were largely directed against DI/II, weakly neutralizing ZIKV, but strongly cross-neutralizing DENV1 and DENV2. However, mAbs from later infection were more diverse in recognition, some potently neutralizing without detectable cross-reactivity with DENV1 and DENV2. Finally, a DIII-specific mAb isolated 106 days after the onset of symptoms provided $100 \%$ protection in mice from lethal ZIKV infection, while a DI/II-specific mAb isolated 4 days failed to do so. Taken together, our study has unveiled the dynamic features of antibody response against ZIKV during natural infection. Potent neutralizing $\mathrm{mAbs}$ identified here provide promising candidates for antibody-based interventions and will also inform epitope-focused vaccine design and development.

\section{Results}

Dynamics of binding and neutralizing antibody response during natural infection. To study the dynamics of antibody response during natural infection, we collected longitudinal blood samples from 2 ZIKV-infected subjects who returned to China from traveling in 2 severely affected South American countries, Venezuela and Surinam. Their infection status was confirmed by reverse transcription PCR (RT-PCR) for ZIKV RNA in multiple serum, saliva, and urine samples (35). Using a commercially available ELISA kit for diagnosis of ZIKV infection and full-length NS1-based ELISA to differentiate ZIKV and DENV1-4 infection (Supplemental Figure 2; supplemental material available online with this article; https://doi. org/10.1172/jci.insight.93042DS1), we showed that the 2 study subjects were positive for ZIKV but not for chikungunya virus, DENV, tick-born encephalitis virus (TBEV), yellow fever virus (YFV), Japanese encephalitis virus (JEV), or West Nile virus (WNV). A total of 5 sequential plasma samples were collected from patient 1 (Pt1) on day 4, 7, 15, 106, and 188, and three from Pt2 on day 6, 12, and 66 after the onset of symptoms. Serial dilutions were made before being applied to the ELISA plate coated with E glycoprotein of ZIKV, DENV1, and DENV2 produced in mammalian 293T cells. The binding activity was detected by anti-human IgG secondary antibody and expressed as optical density (OD) at $450 \mathrm{~nm}$. In Pt1, antibodies became detectable on day 4 , gradually increased on day 7 , and reached the peak on day 15 (Figure 1A, upper panel). During the ensuing days 106 and 188, appreciable decline in antibody titer was noticed but remained fairly high throughout. Interestingly, a similar trend of antibody responses was also found against E glycoprotein of DENV1 and DENV2, suggesting substantial levels of cross-reactive antibodies in ZIKV-infected patients (Figure 1A, upper panel). In Pt2, antibody binding to ZIKV and cross-reactivity to DENV1 and DENV2 followed a similar pattern, although the cross-reactivity to DENV2 appeared to be more profound than to DENV1 (Figure 1A, lower panel). As the patients were infected only by ZIKV without DENV, such substantial levels of cross-reactivity must be due to the sequence and structure shared between ZIKV and DENV (Supplemental Figure 1). In addition, the same set of plasma samples were also evaluated for their neutralizing activity against ZIKV and cross-reactivity against DENV1 

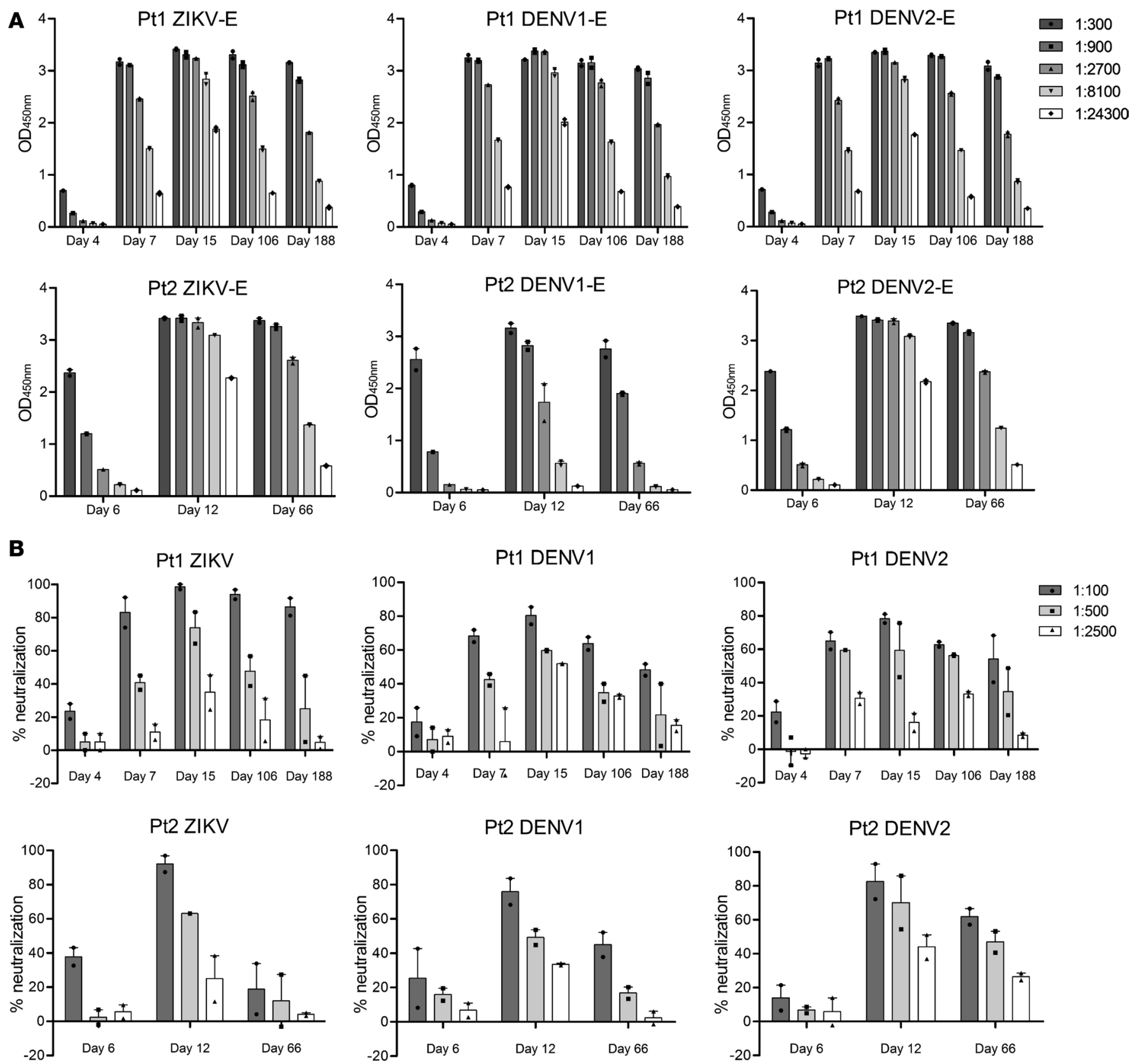

Figure 1. Binding and neutralizing activity of sequential plasma samples from $\mathbf{2}$ ZIKV-infected individuals during natural infection. Serial dilutions of plasma samples were analyzed for their binding (A) to E glycoprotein of ZIKV (GZ01, KU820898), DENV1 (GZ35, KP723476), and DENV2 (GZ05, KP012546) by ELISA and for neutralizing activity (B) against ZIKV (GZ01), DENV1 (Hawaii strain), and DENV2 (New Guinea strain) by plaque assay. All results were derived from at least 2 independent experiments.

and DENV2. Similar to binding antibody, neutralizing antibody also demonstrated comparable dynamics against ZIKV, DENV1, and DENV2, although neutralizing ZIKV was more efficient than crossneutralizing DENV1 and DENV2 (Figure 1B). In Pt2, a precipitous drop in neutralizing activity against ZIKV was found on day 66, although reasonable levels of cross-neutralizing against DENV1 and DENV2 remained detectable (Figure 1B, lower panel).

Domain-specific antibody response during natural infection. We also studied the dynamics of antibody response through domain-specific E glycoprotein expressed on the yeast surface $(15,36-38)$. Yeast clones displaying DI/II or DIII of ZIKV, DENV1, and DENV2 were constructed and incubated with the sequential plasma samples to monitor the changes in antibody specificity over time. In Pt1, DI/II-specific antibodies were barely detectable on day 4 but reached the peak intensity on day 7 , followed by a gradual decline 

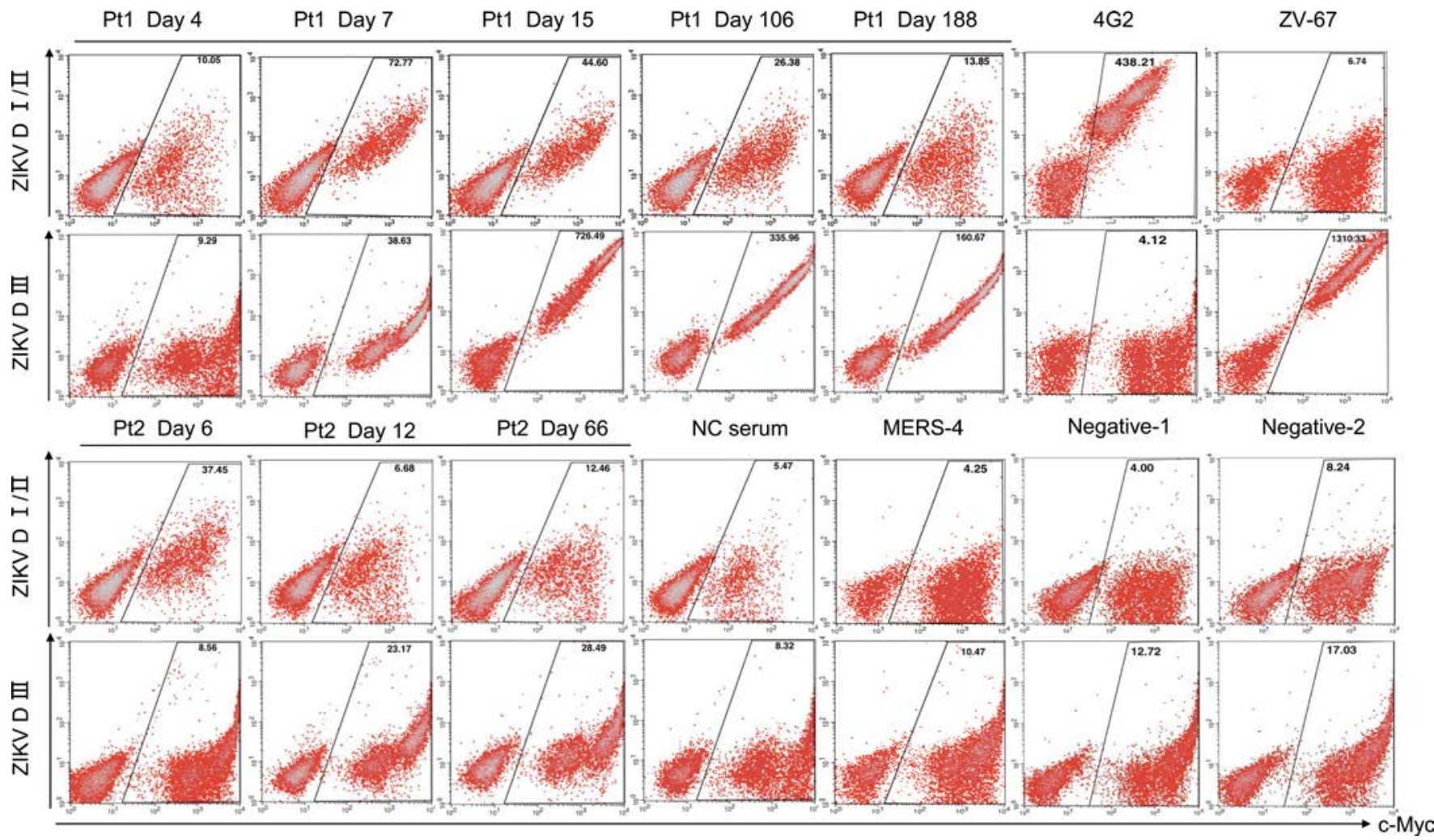

Figure 2. Binding of sequential plasma samples to ZIKV DI/DII- and DIII-expressing yeast clones. Sequential plasma samples (1:100 dilution) were incubated with domain-specific yeast clones and analyzed by FACS. Positive control mAbs 4G2 and ZV-67 were used for specific recognition of DI/II and DIII, respectively. Negative controls included plasma sample from a healthy individual (NC serum), mAb MERS-4 previously isolated against MERS-CoV, and the cells staining without corresponding patients' plasma (Negative-1) or PE labeled anti-human IgC secondary antibody (Negative-2). C-Myc is a protein tag used for monitoring $\mathrm{DI} / \mathrm{Il}$ and $\mathrm{DIII}$ expression under the induction condition.

on day 15 and 106 until diminishing to negligible levels on day 188 (Figure 2, upper panel). DIII-specific antibodies, however, became detectable on day 7, reached the peak on day 15, and persisted into day 106 and 188 despite a noticeable decline in antibody intensity (Figure 2, upper panel). In Pt2, antibody intensity was significantly lower than that in Pt1 against both displayed DI/DII and DIII (Figure 2, lower panel). However, a similar delay in peak intensity for DIII was also found compared with DI/II. Interestingly, when the same set of plasma samples were analyzed against DI/II and DIII of DENV1 and DENV2, the cross-binding was exclusively targeted to DI/II, irrespective of time points during the course of infection (Supplemental Figure 3), suggesting that the cross-binding to E glycoprotein of DENV1 and DENV2 (Figure 1) was largely mediated through the DI/II recognition. In fact, the temporal changes in such cross-binding were highly correlated with the binding to E glycoprotein, as well as neutralizing activity against ZIKV, DENV1, and DENV2 (Figure 1). The peak in cross-binding to DENV DI/II was therefore about 1 week behind the peak of homologous recognition of ZIKV DI/DII, indicating the existence of antibodies with shared as well as differential recognition between DENV DI/II and ZIKV DI/II.

Domain-specific antibody depletion for plasma binding and neutralization. To further confirm DI/II- and DIII-specific plasma antibody activities, we conducted domain-specific antibody depletion experiments. Specifically, plasma samples were absorbed with multiple rounds of ZIKV DI/II- and DIII-specific yeast clones before being analyzed for binding and neutralizing activities. As shown in Supplemental Figure 4A, depletion of ZIKV DI/II-specific binding antibodies resulted in elimination of cross-binding to DI/II of DENV1 and DENV2, while binding to ZIKV DIII remained unaffected. On the other hand, depletion of ZIKV DIII-specific antibodies had a negligible effect on cross-binding to DI/II of DENV1 and DENV2 (Supplemental Figure 4B). These results clearly indicate that DI/II was responsible for most if not all of the cross-binding activities between ZIKV and DENV1-2. Next, we measured the impact of domain-specific depletion on the plasma neutralizing activities. Four sequential plasma samples with substantial neutralizing activities were analyzed before and after absorption. As shown in Figure 3B, increasing contribution of 
A

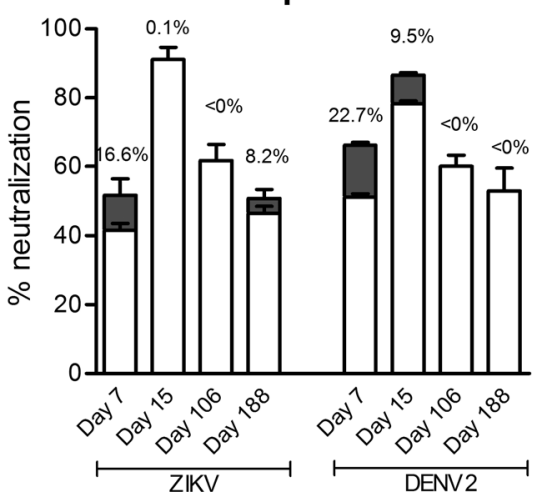

B

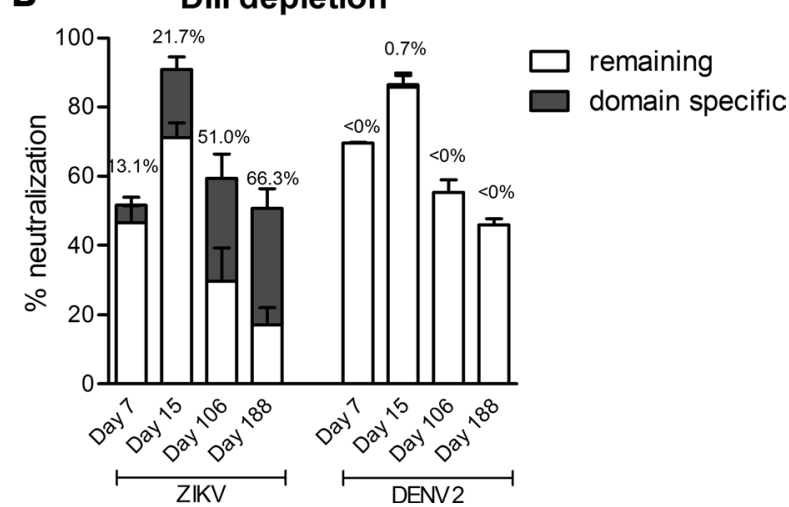

Figure 3. Contribution of DI/II- and DIII-specific antibody to overall neutralization. Comparison of plasma neutralization before and after absorption with yeast clones expressing ZIKV DI/II (A) or DIII (B). The percent contribution was indicated in dark gray, which was calculated by comparing the neutralizing activity of plasma samples that treated with ZIKV DI/II or DIII and irrelevant CD20 yeast clones.

DIII-specific neutralizing activities was found against ZIKV over the course of infection from $13.1 \%$ on day 7 to $21.7 \%$ on day $15,51.0 \%$ on day 106 , and all the way up to $66.3 \%$ on day 188 , while the cross-neutralizing activities against DENV2 were not affected. Interestingly, a sizable portion of neutralizing activities against ZIKV remained, despite DIII depletion, presumably due to those against DI/II and those recognizing more complex quaternary epitopes composed of more than one domain or E glycoprotein. To test this hypothesis, we compared the plasma-neutralizing activities before and after absorption with ZIKV DI/ II-specific yeast clones. To our surprise, despite a complete depletion of ZIKV DI/II binding and DENV1 and DENV2 cross-binding antibodies (Supplemental Figure 4A), neutralizing activities against ZIKV and cross-neutralizing activities against DENV2 remained largely unchanged, in particular for the later samples (Figure 3A). While part of those against ZIKV could be due to the DIII-specific neutralizing antibodies, those cross-neutralizing DENV2 must be arising from those recognizing more complex DI/II epitopes than the ZIKV DI/II-specific yeast clones could express and present. In other words, while binding antibodies to ZIKV DI/II- and DIII-specific yeast clones represent a useful and meaningful biomarker for plasma antibody response, they are by no means complete, in particular when it comes to the neutralizing activities. Additional approaches such as isolation and characterization of monoclonal antibodies will certainly strengthen and compliment the plasma-based profiling.

Isolation and sequence analysis of $m A$ bs from $B$ cells. To further characterize the dynamics of antibody response, we set out to isolate $\mathrm{mAbs}$ from plasmablasts (IgD-IgMCD27 $\mathrm{CD} 38^{\text {high }}$ ) and memory (IgD-IgM-CD2 $27^{+} \mathrm{CD} 38^{\text {low }}$ ) B cells. Greater emphasis was placed on Pt1, due to his stronger antibody responses and availability of a larger number of samples than Pt2. A total of $14 \mathrm{mAbs}$ were successfully obtained, of which 12 were from Pt1 and 2 were from Pt2 (Figure 4A). We first conducted phylogenetic analysis on the amino acid sequences of the antibody variable regions and identified a couple of dominant clusters (Figure 4B). For example, on the tree built upon the heavy chain variable region (left panel), the IGHV4-39 family contained 6 (ZK17F11, ZK22F6, ZK8-4, ZK2B3, ZK12G2, and ZK12-2) while IGHV3-23 had 4 (ZK7C3, ZK21F2, ZK3-1, and ZK5-2) out of the total 14 mAbs isolated. Similar dominance in the IGHV439 family was also found in a separate study (32). Both of these 2 families have the ZIKV DI/II specificity and DENV1 DI/DII and DENV2 DI/DII cross-reactivity (see below). The remaining ZK2B10 and ZK1G4 belonged to the IGHV1-8 family, while ZK2C2 and ZK6F5 belonged to IGHV3-30 and IGHV1-18, respectively. Their binding specificities were much more diverse compared with those in the 2 clusters (see below). On the tree constructed upon the light chain variable region (right panel), antibody clustering was in general mirrored that on the heavy chain variable region with IGKV1-9 and IGKV1-12 corresponding to IGHV4-39 and IGHV3-23 family, respectively. This finding suggested those mAbs within each cluster were derived from the common antibody ancestors. It is interesting to note that mAbs isolated early during infection appeared to be more divergent from the germline sequence than the later mAbs (Figure 4A). More thorough studies would be required to further validate this observation, ideally in the context of specific antigens or even specific epitopes. Finally, except for ZK2C2, all mAbs had longer CDR3 regions in the heavy chain than the 


\begin{tabular}{|c|c|c|c|c|c|c|c|c|c|c|c|c|c|c|c|c|c|c|}
\hline \multirow{2}{*}{$\mathrm{mAb}$} & \multirow{2}{*}{ Origin } & \multirow{2}{*}{ Day } & \multirow{2}{*}{$\mathrm{P} / \mathrm{M}$} & \multicolumn{3}{|c|}{ Epitope } & \multicolumn{3}{|c|}{ IC50(ug/ml) } & \multicolumn{3}{|c|}{ EC5O(ng/ml) } & \multicolumn{2}{|r|}{ Heavy chain } & \multirow[b]{2}{*}{$\%$} & \multicolumn{3}{|c|}{ Light chain } \\
\hline & & & & ZIKV & DENV1 & DENV2 & ZIKV & DENV1 & DENV2 & ZIKV & DENV & DENV2 & V family & CDR3 sequence & & V family & CDR3 sequence & $\%$ \\
\hline$\overline{Z K 8-4}$ & Pt1 & 4 & $P$ & $\mathrm{DI} / \mathrm{II}$ & $\mathrm{DI} / \mathrm{II}$ & $\mathrm{DI} / \mathrm{II}$ & $>40$ & 0.67 & 2.10 & 9.7 & 7.8 & 8.1 & IGHV4-39 & 9 VRQEKNWFDS & 88.5 & IGKV1-9 & QHLSNYLFT & 92.9 \\
\hline ZK3-1 & Pt1 & 7 & $P$ & $\mathrm{DI} / \mathrm{II}$ & $\mathrm{DI} / \mathrm{II}$ & $\mathrm{DI} / \mathrm{II}$ & 10.47 & 0.17 & 0.77 & 44.9 & 18.7 & 36.8 & IGHV3-23 & 3 ANTIWSADSGGSVGAFHS & 89.8 & IGKV1-12 & QQASSFPWT & 92.6 \\
\hline ZK5-2 & Pt1 & 7 & $P$ & $\mathrm{DI} / \mathrm{II}$ & $\mathrm{DI} / \mathrm{II}$ & $\mathrm{DI} / \mathrm{II}$ & 13.49 & 0.30 & 1.77 & 27.4 & 11.9 & 20.8 & IGHV3-23 & 3 ANTIWSVDSGGSVGAFHY & 94.2 & IGKV1-12 & QQANSFPWT & 96.5 \\
\hline ZK21F2 & Pt1 & 15 & M & DI/II & $\mathrm{DI} / \mathrm{II}$ & $\mathrm{DI} / \mathrm{II}$ & 4.27 & 0.42 & 1.84 & 237.3 & 180.0 & 192.5 & IGHV3-23 & 3 TNPVWSADSGGSVGAFHH & 89.1 & IGKV1-12 & QQANSFPWT & 90.8 \\
\hline ZK2B3 & Pt1 & 15 & M & DI/II & $\mathrm{DI} / \mathrm{II}$ & $\mathrm{DI} / \mathrm{II}$ & 6.13 & 0.53 & 4.78 & 9.0 & 8.0 & 9.0 & IGHV4-39 & 9 ARQFGNYFDY & 91.9 & IGKV1-9 & QHLSNYLFI & 94.3 \\
\hline ZK12G2 & Pt1 & 15 & M & $\mathrm{DI} / \mathrm{II}$ & $\mathrm{DI} / \mathrm{II}$ & $\mathrm{DI} / \mathrm{II}$ & 9.86 & 0.07 & 1.02 & 5.3 & 5.2 & 4.6 & IGHV4-39 & 9 ARQFGNYFNS & 91.3 & IGKV1-9 & QLLSNYLFT & 93.6 \\
\hline ZK17F11 & Pt1 & 15 & M & $\mathrm{DI} / \mathrm{II}$ & $\mathrm{DI} / \mathrm{II}$ & $\mathrm{DI} / \mathrm{II}$ & 13.18 & 0.48 & 7.12 & 9.7 & 7.3 & 7.4 & IGHV4-39 & 9 ARQLGNYFNY & 91.0 & IGKV1-9 & QHLSNYFFT & 94.3 \\
\hline ZK22F6 & Pt1 & 15 & M & $\mathrm{DI} / \mathrm{II}$ & $\mathrm{DI} / / \mathrm{I}$ & $\mathrm{DI} / \mathrm{II}$ & 1.56 & 0.08 & 0.06 & 6.1 & 6.5 & 5.7 & IGHV4-39 & 9 ARQFGNFFDY & 91.2 & IGKV1-9 & QVLSDYQFT & 92.2 \\
\hline ZK2B10 & Pt1 & 106 & M & DIII & - & - & 0.04 & $>40$ & $>40$ & 8.4 & $>200$ & $>200$ & IGHV1-8 & ARVRSGTNYGSYYYYYGMDV & 92.3 & IGLV1-47 & ASWDDSLSGHWV & 97.3 \\
\hline ZK7C3 & Pt1 & 106 & M & $\mathrm{DI} / \mathrm{II}$ & - & - & 0.11 & $>40$ & $>40$ & 22.0 & $>200$ & $>200$ & IGHV3-23 & 3 AKCQQFVGDDAFDI & 95.9 & IGLV3-21 & QVWYSTSDHVV & 96.1 \\
\hline ZK2C2 & Pt1 & 106 & M & DI/IIIII & - & - & 3.86 & $>40$ & $>40$ & 24.9 & $>2000$ & $>2000$ & IGHV & AKGHLID & 96.3 & IGLV & AAWDDSLNGWL & 96.6 \\
\hline ZK6F5 & Pt1 & 106 & M & $\mathrm{DI} / \mathrm{II}$ & . & - & $>40$ & $>40$ & $>40$ & 35.6 & 220.9 & 78.8 & IGHV1-18 & 3 ARSGYSSAARGGWFDP & 96.6 & IGKV3-11 & QQRANWPLT & 98.9 \\
\hline ZK12-2 & Pt2 & 10 & $P$ & $\mathrm{DI} / \mathrm{II}$ & $\overline{D I} / / \mathrm{II}$ & $\overline{D I} / / \mathrm{I}$ & 12.59 & 0.22 & 0.41 & 4.8 & 4.4 & 4.5 & IGHV4-39 & 9 ARQSASTGTMAVGSFDY & 89.6 & IGKV1-12 & QQSGAFPWT & 92.6 \\
\hline ZK1G4 & Pt2 & 12 & M & DI/IIIII & $\mathrm{DI} / \mathrm{II}$ & $\mathrm{DI} / \mathrm{II}$ & 6.31 & 1.80 & 2.83 & 331.2 & 205.9 & 272.6 & IGHV1-8 & FVVVIPGREQFFDY & 93.8 & IGLV3-1 & QAWDSRT & 94.3 \\
\hline
\end{tabular}
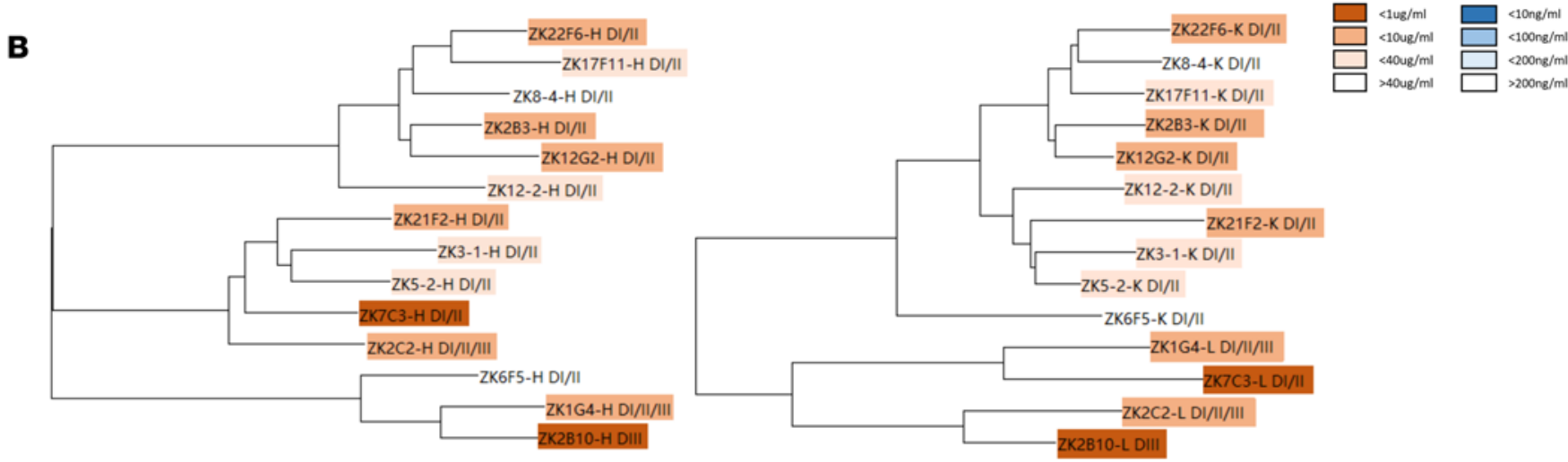

$\longmapsto 0000$

$\longmapsto 0050$

Figure 4. Summary of isolated mAbs and phylogenetic analysis. (A) Antibodies were listed accordingly to the sampling time points from the patients together with the type of B cells originally derived from, P for plasmablast and $\mathrm{M}$ for memory. Epitope specificity was determined by staining with DI/II- and DIII-expressing yeast clones from ZIKV, DENV1 and DENV2. IC ${ }_{50}$ represents the half-maximal inhibitory concentrations in the plaque neutralization assay, whereas $\mathrm{EC}_{50}$ represents the half-maximal effective concentrations in ELISA binding assay, and both of which are presented in heatmap format for differential activities. Only the CDR3 regions of heavy and light variable chain are shown together with their family names and percent similarity with the corresponding germline ancestor sequence. (B) Unrooted neighbor-joining tree depicting the relationship of isolated mAbs; left panel for the heavy chain and right panel for the light chain variable region. The branch length is drawn to scale so that the relatedness between different amino acid sequences can be readily assessed. Individual sequences are named at the tip of the branches, and their epitope specificity and neutralizing potency are indicated as in the heatmap.

corresponding light chain. The length of CDR3 for the heavy chain ranged from 7 (ZK2C2) to 20 amino acids (ZK2B10), while that for the light chain ranged from 7 (ZK1G4) to 12 amino acids (ZK2B10).

Binding and neutralization activities of isolated $m A b s$. We next measured their binding to the E glycoprotein of ZIKV, DENV1, and DENV2 produced in mammalian 293T cells by ELISA. Among the $14 \mathrm{mAbs}, 3$ (ZK2B10, ZK7C3, and ZK2C2) were ZIKV E glycoprotein specific, while the remaining 11 cross-reacted with the E glycoprotein of DENV1 and DENV2 (Figure 5A and Figure 4A). ZK6F5 had fairly weak cross-binding compared with the rest. Control mAbs C8 and ZV67 previously reported by others demonstrated expected specificity $(16,17)$. These $\mathrm{mAbs}$ were then subjected to a plaque reduction neutralization test against ZIKV, DENV1, and DENV2 (Figure 5B and Figure 4A). The most potent neutralizing mAbs against ZIKV were 2 of the 3 specific binders to ZIKV E glycoprotein (ZK2B10 and ZK7C3) with the half-maximal inhibitory concentrations $\left(\mathrm{IC}_{50}\right.$ ) of 0.04 and $0.11 \mu \mathrm{g} / \mathrm{ml}$, respectively. The remaining $\mathrm{mAbs}$ showed only modest (1.56 to $13.49 \mu \mathrm{g} / \mathrm{ml})$ or even no $\left(\mathrm{IC}_{50}>40 \mu \mathrm{g} / \mathrm{ml}\right)$ neutralizing activity. Strikingly, all these mAbs except ZK2C2 and ZK6F5 demonstrated potent cross-neutralizing activity against DENV1 and DENV2 with $\mathrm{IC}_{50}$ ranging from 0.06 to $4.78 \mu \mathrm{g} / \mathrm{ml}$, comparable with those isolated from DENV-infected patients $(15,19,39)$. ZK6F5 failed to show any cross neutralization against DENV1 and DENV2. In addition, such cross neutralization appeared to be more effective against DENV1 than DENV2 with average $\mathrm{IC}_{50} 0.34 \mu \mathrm{g} / \mathrm{ml}$ for the former and $2.43 \mu \mathrm{g} / \mathrm{ml}$ for the latter. It is interesting to note that the 2 potent neutralizing mAbs (ZK2B10 and ZK7C3) were derived from memory B cells collected on day 106 after the onset of symptoms, whereas almost all antibodies that were poorly neutralizing and cross-reacted with DENV1 and DEVN2 were from the plasmablasts or memory B cells in the preceding samples (Figure $5 \mathrm{~B}$ and Figure $4 \mathrm{~A}$ ). As previously shown, control mAb C8 demonstrated impressive cross-neutralizing activity (16), whereas ZV67 was specifically neutralizing ZIKV (17). 
$\mathbf{A}_{4} \quad$ zK8-4
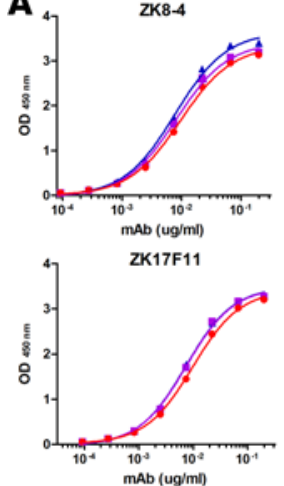

ZK12-2

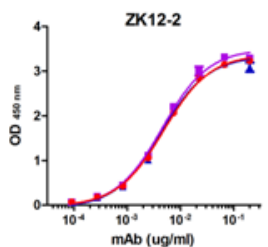

B
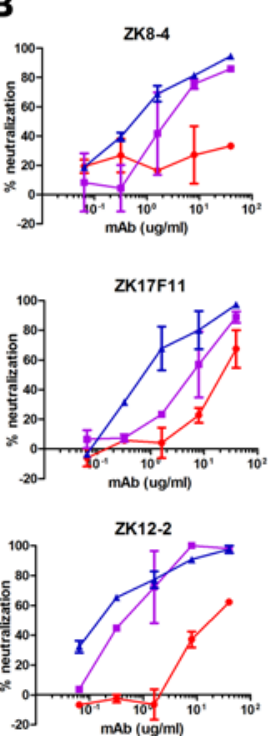
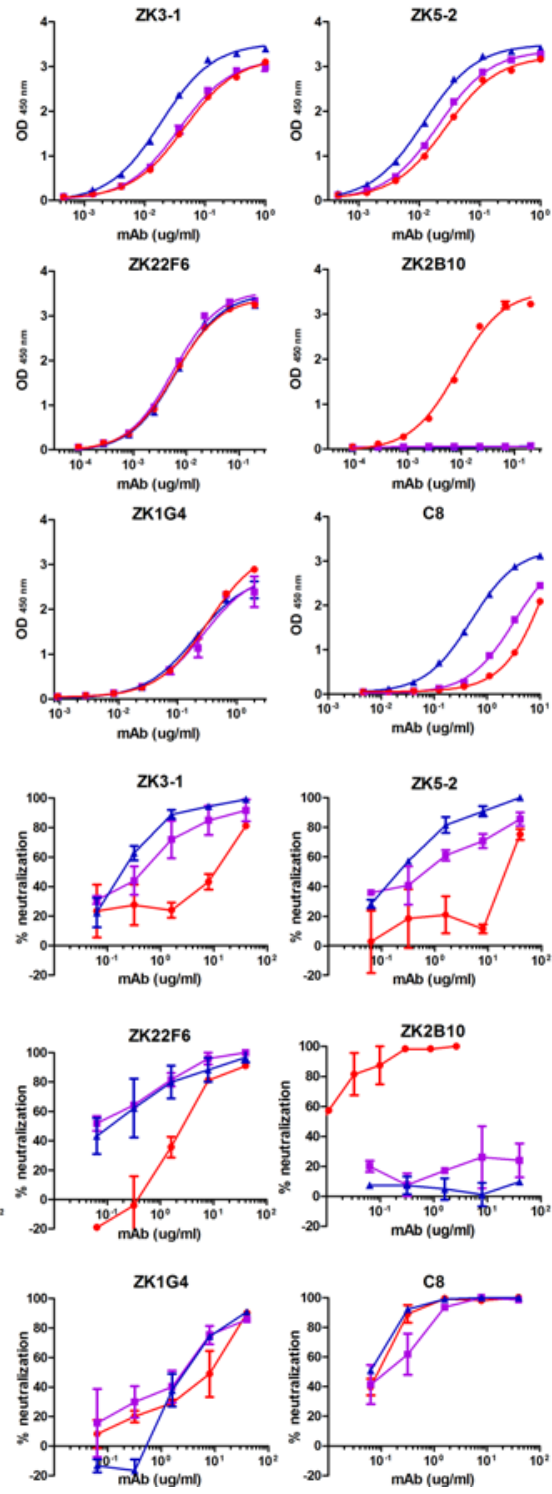

ZK1G4
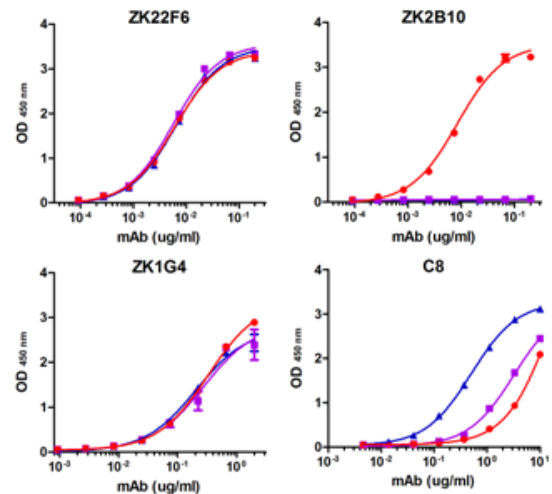

C8
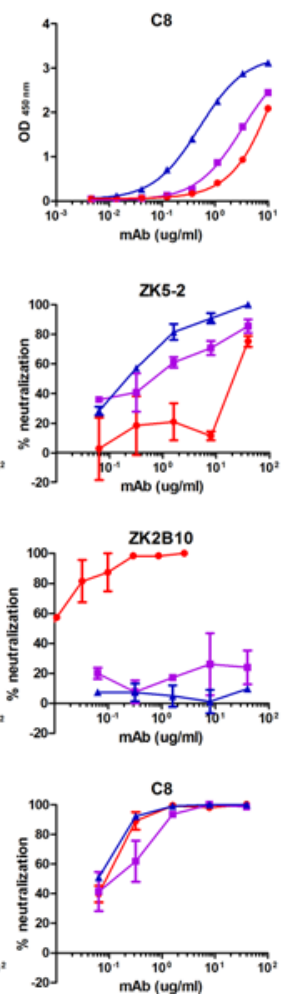
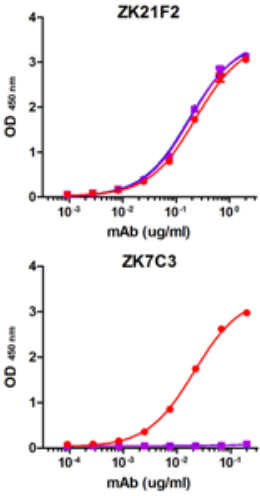

ZV-67
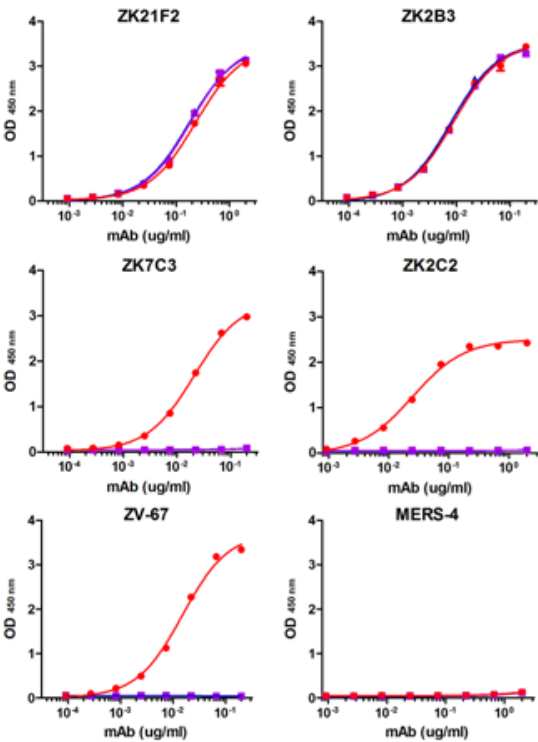

MERS-4
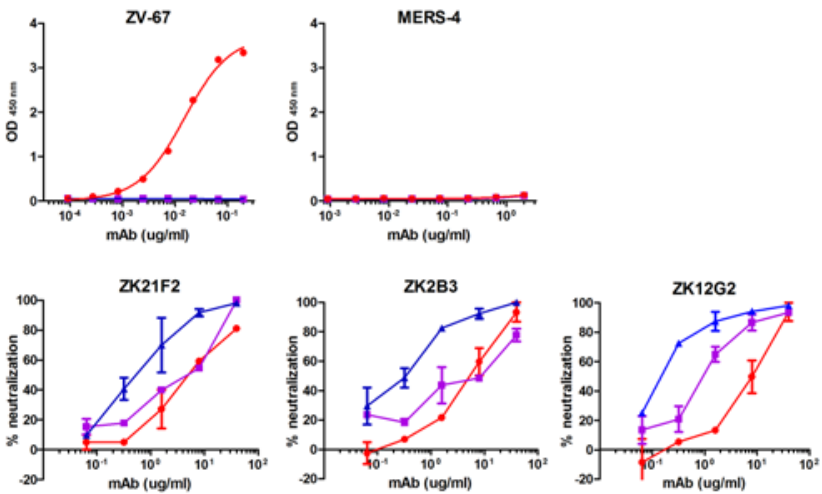

ZK6F5
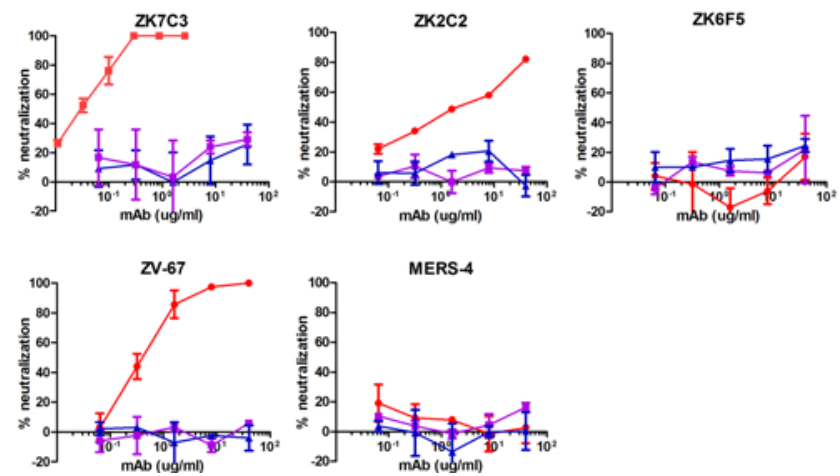

Figure 5. Binding and neutralizing activity of isolated mAbs from ZIKV-infected individuals. Twelve mAbs from Pt1 and $2 \mathrm{mAbs}$ from Pt2 were serially diluted and analyzed for their binding (A) to E glycoprotein of ZIKV (GZ01, KU820898), DENV1 (CZ35, KP723476), and DENV2 (CZ05, KP012546) by ELISA and for neutralizing activity (B) against ZIKV (GZ01), DENV1 (Hawaii strain), and DENV2 (New Guinea strain) by plaque assay. All results were derived from at least 2 independent experiments. Positive control mAbs C8 and ZV-67 were used for cross-neutralizing and ZIKV-specific mAb, respectively. Negative control mAb MERS-4 was previously isolated against MERS-CoV.

Epitope mapping of isolated mAbs. We used two methods to determine the epitope specificity of isolated mAbs. One was through binding to yeast clones displaying the DI/II or DIII of ZIKV, DENV1, and DENV2, and the other was through pairwise competition measured using surface plasmon resonance (SPR). Based on the unequivocal binding pattern shown in Figure 6, the epitope specificities of isolated mAbs can be broadly classified into 3 major categories DI/DII, DIII, and DI/DII/DIII (Figure 4A). In Pt1, all mAbs isolated from the first 15 days, such as ZK8-4, ZK3-1, ZK5-2, ZK21F2, ZK2B3, ZK12G2, ZK17F11, and ZK22F6, fell into the DI/DII category (Figure 6 and Figure 4A). Four mAbs from day 106 were rather divergent in their epitope specificities. ZK7C3 and ZK6F5 belonged to DI/DII, ZK2B10 belonged to DIII, while ZK2C2 belonged to DI/DII/DIII. In Pt2, ZK12-2 was in DI/DII while ZK1G4 was in DI/DII/DIII. Furthermore, all mAbs in the ZIKV DI/DII category strongly cross-reacted with the DI/DII of DENV1 and DENV2, although ZK7C3, ZK2C2, and ZK6F5 had only marginal but detectable cross-reactivity with DI/DII of DENV2 for reasons of which we are currently uncertain (Supplemental Figure 5, A and B). 

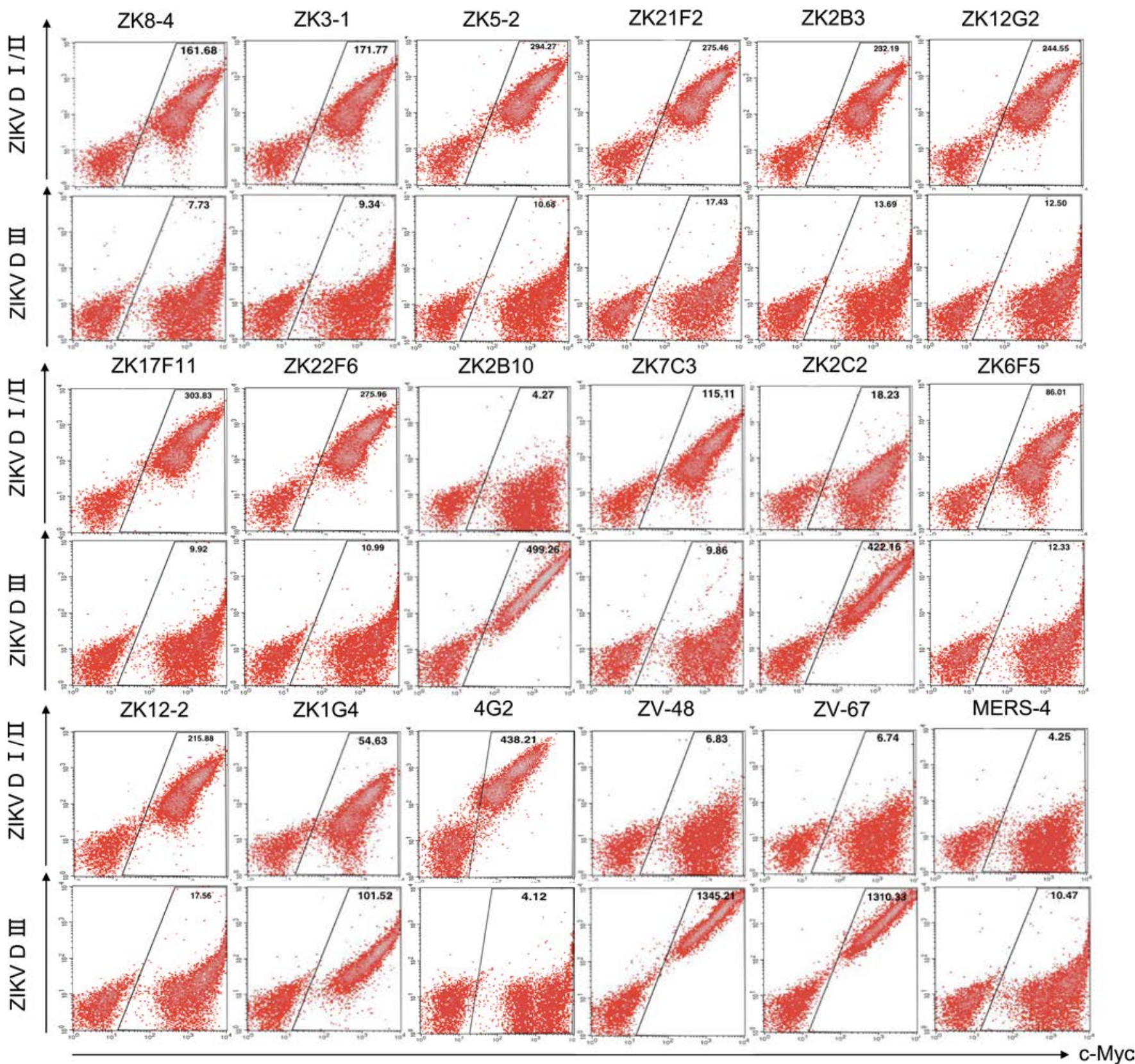

Figure 6. Epitope mapping by ZIKV DI/DII- and DIII-expressing yeast clones. Each mAb $(10 \mu \mathrm{g} / \mathrm{ml})$ were incubated with domain-specific yeast clones and analyzed by FACS. Positive control mAbs 4G2, ZV48, and ZV-67 were used for specific recognition of DI/II and DIII. Negative control mAb MERS-4 was previously isolated against MERS-CoV. C-Myc is a protein tag used for monitoring DI/II and DIII expression under induction condition.

ZK2B10 had no detectable cross-reactivity with either DENV1 or DENV2. Such high degree of cross-recognition at the $\mathrm{mAb}$ level may provide some explanations for the strong cross-reactivity found in the polyclonal plasma samples (Figure 1 and Supplemental Figure 3). We next confirmed the epitope and determined their spacial relationship through pairwise competition using SPR. Specifically, representative mAbs from each category, DI/DII, DIII, and DI/DII/DIII, were selected based on their clustering on the phylogenetic tree and sequence similarity in the heavy and light chain CDR3 regions (Figure 4). The selected mAbs within each category were then paired and sequentially applied to the captured ZIKV E glycoprotein on a CM5 sensor chip to monitor for additional binding to determine whether the $2 \mathrm{mAbs}$ recognized the separate or closely situated epitopes. As shown and summarized in Supplemental Figure 6, our isolated mAbs occupied fairly diverse and distinct epitopes. Among those in category DI/DII, ZK8-4, ZK3-1, ZK17F11, and ZK12-2 recognized similar and overlapping epitopes which were distinct from those recognized by ZK6F5 and 
A

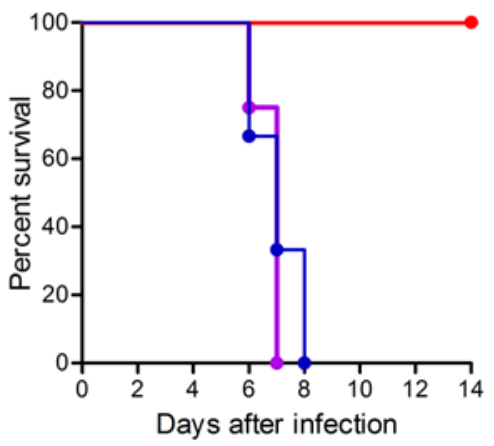

B

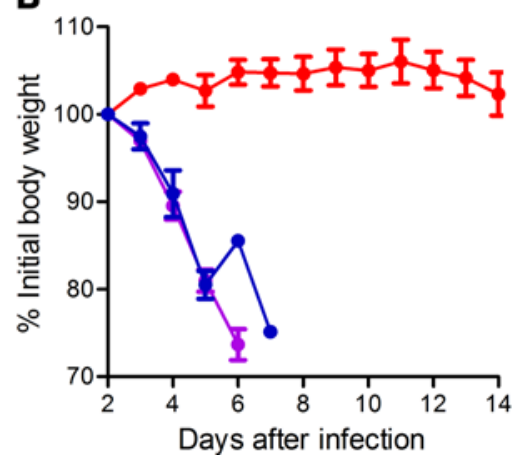

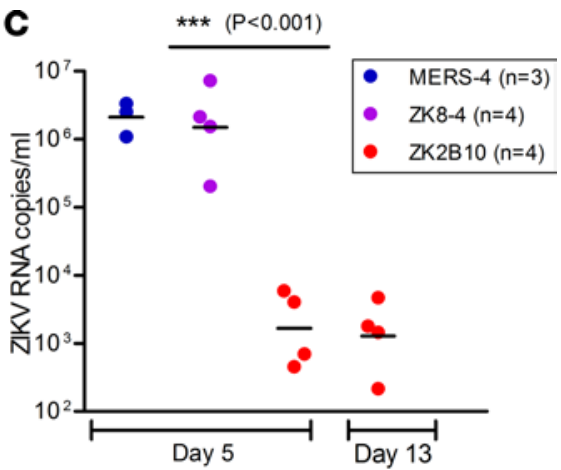

Figure 7. Protective activities of mAbs in AG6 mice. One day after challenge with $1 \times 10^{4}$ pfu of ZIKV GZ01, AG6 mice were treated with $300 \mu g$ mAbs ZK2B10, ZK8-4, or control mAb MERS-4 i.p. and monitored for survival (A), body weight (B) and blood ZIKV RNA (C) up to 14 days. ZK2B10 provided $100 \%$ protection against ZIKV diseases, while ZK8-4 and the control MERS-4 failed ( ${ }^{* *} P<0.001$, one-way ANOVA) with an average time of death of 7 and 8 days. The body weight and ZIKV RNA in the whole blood derived from a single measurement showed distinct results among the study groups. The number of animals used in each group $(n)$ is also indicated.

ZK7C3 (Supplemental Figure 6A). In the category DIII, ZK2B10 competed with a positive control ZV-67 but not ZV-48, suggesting its epitope is located in the lateral ridge region within DIII (17). Furthermore, no competition was found between ZK1G4 and ZK2C2 in the category DI/DII/DIII. However, ZK1G4 competed with 5 out of the 6 representative mAbs in the category DI/DII, while ZK2C2 competed with all the 3 listed in the DIII (Supplemental Figure 6A). These results confirmed the epitopes of ZK1G4 and ZK2C2 were located in the juncture region between DI/DII and DIII, with ZK1G4 leaning more toward D1/DII while ZK2C2 more toward DIII. The exact epitope of these mAbs would require future structural and mutagenesis studies.

Protective efficacy of ZK8-4 and ZK2B10 in mice. Among the isolated mAbs, we selected ZK8-4 and ZK2B10 to test their protective efficacy in vivo largely due to their distinct biologic and genetic features and associated time points when they were initially isolated. As shown above, ZK8-4 is DI/DII cross-reactive, poorly neutralizing, and isolated from a plasmablast B cell on day 4, whereas ZK2B10 is ZIKV DIII specific, potent neutralizing, and isolated from the memory B cells on day 106 after the onset of symptoms. To determine whether these distinct features could result in differential protective efficacy in vivo, we tested them in a mouse model deficient in IFN receptors and signaling $(40,41)$. Specifically, 4- to 6-week-old C57BL $/ 6$ mice deficient in IFN $\alpha,-\beta$, and $-\gamma$ receptors (AG6 mouse) were challenged with $1 \times 10^{4}$ pfu ZIKV (GZ01 strain) via the i.p. route. One day after challenge, a single dose of $300 \mu \mathrm{g}$ ZK8-4, ZK2B10, or negative-control $\mathrm{mAb}$ against Middle East respiratory syndrome coronavirus (MERS-CoV) were administered via i.p. On day 5 and day 13 after challenge, blood samples were collected from each animal for viral load measurement. All animals were monitored for signs of disease, survival, and weight change for 14 days. As shown in Figure 7A, animals treated with ZK8-4 and negative-control MERS-4 succumbed to ZIKV infection with an average time of death of 7 and 8 days, respectively. Severe weight loss was also found during the same period (Figure 7B). In contrast, ZK2B10 treatment afforded 100\% protection and associated with increased body weight over 14 days of observation. ZIKV RNA load in the blood was significantly higher in ZK8-4- or MERS-4- than ZK2B10-treated animals (Figure 7C). These results clearly demonstrate that potent neutralizing ZK2B10 rather than poorly neutralizing ZK8-4 can protect animals from diseases and may play a protective role in the study subject during natural infection.

\section{Discussion}

We report here the kinetics of antibody responses against ZIKV during natural infection in 2 convalescent individuals. The uniqueness of our study lies in the longitudinal approach to characterize and relate polyclonal with $\mathrm{mAb}$ responses from the same individuals. At the polyclonal antibody level, we showed the rapid responses in binding and neutralizing activities peaked about 2 weeks and was sustained up to 188 days after the onset of symptoms. A substantial degree of cross-reactivity with DENV1 and DENV2 was found throughout the study. By decomposing the binding response into DI/DII and DIII of the E glycoprotein, we showed antibody development in humans followed a spatiotemporal hierarchy: DI/II 
peaked and waned during early infection, but DIII persisted for an extended period of time. A substantial degree of cross-reactivity with DENV1 and DENV2 were predominantly mediated through D1/DII but not DIII. A clear trend of increase in DIII-specific neutralizing activity was observed over the course of infection, although a substantial portion of neutralizing activities was not accounted for. Antibodies that recognized more complex quaternary epitopes composed of more than one domain or E glycoprotein may offer some plausible explanations. At the mAb level, we showed the mAbs isolated during the first 2 weeks after the onset of symptoms were largely targeted to DI/II, weakly neutralizing, and broadly cross-reactive with DENV1 and DENV2. In contrast, the mAbs isolated about 3 months later expanded their specificity to include DI/DII, DIII, and DI/DII/DIII, some of which were potently neutralizing and ZIKV specific. Competitive binding measured by SPR also indicated the isolated mAbs occupied diverse and distinct epitopes covering substantial surfaces of the E glycoprotein. It is interesting to note that mAbs isolated early during infection appeared to be more divergent from the germline sequence than the later mAbs, and more thorough studies would be required to further validate this observation. Finally, the potent but not weak neutralizing $\mathrm{mAbs}$ provided $100 \%$ protection in mice from lethal ZIKV infection. Taken together, the dynamic features unveiled here will assist us for a better understanding the pathogenesis of ZIKV infection. Potent neutralizing mAbs identified here provide promising candidates for antibody-based interventions and also inform epitope-focused vaccine design and development.

We believe our study is the first in the field to delineate the longitudinal antibody response against ZIKV from both polyclonal and monoclonal levels. The distinct dynamic and biological features associated with domain-specific antibody response are quite unique and perfectly in line with those reported in DENV infections $(42,43)$. It is well documented that people exposed to DENV primary infection demonstrated a transient period of broad protection against heterologous serotype. The long-term protection, however, appeared relatively later but more potent and specific to the infecting homologous serotype (43-47). Given what we have identified here, we could hypothesize those transient and broad antibody responses are likely mediated through DI/DII, while those in the long-term protection are likely mediated through broader scope in specificity. Our mAbs isolated from ZIKV-infected individuals and others from DENV-infected individuals strongly support this hypothesis (31). ZIKV DI/DII-specific mAbs are predominant during early infection, are weakly neutralizing, and are broadly cross-reactive with DI/DII of DENV1 and DENV2. In fact, many of these mAbs can cross-neutralize DENV1 and DENV2 more effectively than ZIKV, suggesting the same epitope may be differentially exposed and more accessible in a DENV than ZIKV context. On the other hand, mAbs isolated from DENV infection with DI/DII specificity also demonstrated strong cross-reactivity and protection against ZIKV $(16,46,48)$. Furthermore, analysis of sera from individuals with acute DENV infection showed a substantial capacity to bind and neutralize ZIKV $(43,45)$, mirroring what we found in ZIKV-infected patient responses to DENV. At the current stage, the roles of these early and cross-reactive DI/DII-specific mAbs in vivo are uncertain and could be a double-edged sword. Cross-reactivity could certainly provide cross-protection across ZIKV and DENV as observed in previous studies $(30,43,48)$. If this is the case, future therapeutics and vaccines would need to take such advantage to develop universal strategies against ZIKV, DENV, and perhaps other members in the flavivirus family. But cross-reactivity could also result in cross-enhancement and trigger more severe diseases in humans. Indeed, many of the DI/DII mAbs, including ours, demonstrated antibody-dependent enhancement (ADE) in cell culture (Supplemental Figure 6) and in the animal models of ZIKV and DENV infection $(31,45,49,50)$. Whether such enhancement could occur or exacerbate diseases in humans would require future studies, in particular when ZIKV and DENV continue to cocirculate in more geographic regions around the globe. Should such cross-enhancement indeed occur in humans, our current vaccine strategies would have to minimize or completely eliminate the DI/DII and would have to target DIII or related regions instead.

The potent neutralizing mAbs (ZK2B10 and ZK7C3) isolated about 3 months after the onset of symptoms are strictly ZIKV specific and cover distinct epitopes not merely confined to DIII, but also expanded to DI/II or more complex DI/DII/DIII. While their exact epitopes are being actively investigated, it is reasonable to hypothesize that they recognize more complex structures than those isolated earlier in the infection as they cross-competed with mAbs from multiple categories (Supplemental Figure 6). Previous studies of ZIKV and DENV infection have identified potent neutralizing mAbs targeting DIII or quaternary epitopes on the E glycoprotein capable of providing protection against ZIKV infection, disease, and maternal-fetal transmission in a mouse model. One of the potent neutralizing mAbs identified here 
(ZK2B10) also afforded 100\% protection in mice from lethal ZIKV infection, while the poorly neutralizing and DI/DII cross-reactive ZK8-4 isolated on day 4 after the onset of symptoms failed to do so. These results highlight the importance of potently neutralizing rather than weakly or nonneutralizing $\mathrm{mAbs}$ in providing protection against ZIKV infection. Future vaccine strategies would have to focus on inducing this class of antibodies to provide effective and durable protection. However, as those DI/DII-specific mAbs, this class of mAbs, including ours (Supplemental Figure 7), also demonstrated ADE at suboptimal levels in vitro $(17,31)$. Development of ZIKV vaccine would therefore be likely to encounter similar problems facing DENV vaccine in eliciting suboptimal immune responses that might lead to ADE and severe disease. In light of this recognition, prophylactic and therapeutic intervention with potently neutralizing mAbs may offer an alternative for the prevention and treatment of ZIKV, DENV, and perhaps other members in the flavivirus family. In this regard, the fully human mAbs isolated from convalescent humans by others and us represent promising alternatives, as they are generated during a natural infection and are expected to have minimal antigenicity and autoreactivity in humans. To ensure safety and efficacy, future engineering of $\mathrm{mAbs}$ to eliminate the $\mathrm{ADE}$ and selection of $\mathrm{mAb}$ combination with synergistic effects would certainly be a preferred strategy. Finally, it needs to be noted that our study is by no means perfect. To generalize our observation, we would need to increase the number of study subjects and follow an even longer time period after infection. Also, as the DI/DII and DIII expressed on the surface of yeast are expected to deviate from the conformation of native E glycoprotein on the intact virion, we have to be cautious when interpreting the data. Nevertheless, the comprehensive approach used here has provided additional insights into the complex and dynamic features of antibody response against ZIKV during natural infection. Information obtained will improve our understanding of the pathogenesis of ZIKV infection and potential interplay with DENV and other flaviviruses alike. The potent neutralizing mAbs identified here will provide promising candidates for antibody-based intervention and will also assist our rational design of epitope-focused vaccines that will empower the strengths while minimizing the weaknesses of human immune recognition.

\section{Methods}

Patients and blood samples. During the widespread epidemic of ZIKV infection in the Americas in 2016, 2 infected Chinese people returned to the southern metropolitan city Guangzhou, China, from the 2 severely affected South American countries, Venezuela and Surinam. They were hospitalized in the Guangzhou Eighth People's Hospital, and their infection status was confirmed by RT-PCR positivity for ZIKV RNA in multiple serum, saliva, and urine samples (35). Their flavivirus immune status was analyzed by a commercially available kit for diagnosis of ZIKV infection (EUROIMMUN) and by a custom-based ELISA built upon the full-length NS1 proteins derived from ZIKV and DENV1-4 isolated from China (see below). The plasma samples from RT-PCR-confirmed DENV1-infected individual were included for comparison. Pt1 was a 28-year-old man returning from Venezuela in February, 2016, and Pt2 was a 40-year-old woman returning from Suriname in July, 2016. Both patients manifested relatively mild symptoms, including fever, rash, sore throat, and fatigue, and they recovered and were discharged approximately 3 weeks after onset of symptoms (35). The major clinical events, virus load in serum and urine, and kinetics of IgM and IgG antibody responses in Pt1 have previously been reported (35). Persistent high levels of ZIKV load in the urine were found during the first 2 weeks after the onset of symptoms, from which a novel ZIKV strain, GZ01, was successfully isolated. Phylogenetic analysis of the complete coding sequences indicated GZ01 (GenBank number KU820898) was tightly clustered with those circulating in the Americas and belonged to the Asian lineage, including those identified from French Polynesia in 2013 (51). Sequence analysis of ZIKV RNA from Pt2 showed it was identical with Pt1 in both E and NS1 regions and therefore fell into the same Asian lineage. During hospitalization and follow-up visits, a total of 5 sequential blood samples were collected from Pt1 on days 4, 7, 15, 106, and 188, while 3 were collected from Pt2 on days 6, 12, and 66 after the onset of the symptoms. Collected blood samples were separated into plasma and peripheral blood mononuclear cells (PBMCs) by centrifugation through a Ficoll-Hypaque gradient (GE Healthcare). All plasma samples were heat inactivated at $55^{\circ} \mathrm{C}$ for 1 hour before being stored at $-80^{\circ} \mathrm{C}$, while PBMCs were maintained in freezing media and stored in liquid nitrogen until use.

Recombinant ZIKV and DENV E glycoprotein and ELISA analysis. Recombinant E glycoprotein of ZIKV (GZ01, KU820898), DENV1 (GZ35, KP723476), and DENV2 (GZ05, KP012546) were produced in the mammalian 293T cell expression system with a D7-tag at the C-terminus for detection and purification (52). The coding sequences for the ectodomain of ZIKV, DENV1, and DENV2 E glycoprotein were obtained by 
direct RT-PCR amplification of viral RNA derived from either plasma samples or virus stock of infected Chinese patients and cloned into the expression vector pCDNA3.1 (Invitrogen). Approximately 4-5 days after transfection, the recombinant E glycoprotein in the culture supernatant was captured onto the ELISA plate through binding to D7-tag by precoated antibody D7324 (Cliniqa) (52). The captured recombinant E glycoprotein was then used to evaluate plasma levels of antibodies from ZIKV-infected patients, screen for the presence of antibody in the culture supernatant of memory B cells, and measure binding specificities of isolated mAbs from the patients. Specifically, serial dilutions of inactivated plasma samples and isolated mAbs from ZIKV-infected patients were applied to the captured E glycoprotein of ZIKV, DENV1, and DENV2. The binding activity was detected by anti-human IgG conjugated with horseradish peroxidase (Promega) and 3,3',5,5'-Tetramethylbenzidine (TMB) substrate (CWBio).

Full-length ZIKV and DENV1-4 NS1 protein and ELISA analysis. Full-length NS1 proteins of ZIKV (GZ01, KU820898), DENV1 (GZ35, KP723476), DENV2 (GZ05, KP012546), DENV3 (YN02, KF824903), and DENV4 (GZ30, JQ822247) were produced according to the method described above with a D7-tag at the C-terminus. For ELISA analysis, the NS1 in the culture supernatant from transfected 293T cells was captured onto the ELISA plate by precoated anti-D7 antibody. The sequential plasma samples from Pt1 and Pt2 were serially diluted and applied to the NS1-cpatured ELISA plate before the binding activity was detected by anti-human IgG labeled with HRP and TMB substrate. The serial diluted plasma from a DENV1-infected patient was used as positive control.

Isolation of ZIKV human mAbs from plasmablasts and memory $B$ cells. The PBMCs stored in liquid nitrogen was thawed in $37^{\circ} \mathrm{C}$ before being stained with a mixture of fluorescence-labeled monoclonal antibodies for various surface makers (IgD-FITC, CD19-ECD, CD27-PC7, CD38-APCA750, IgM-PB, and CD45-KO; Beckman Coulter) to distinguish plasmablasts (IgD-IgM-CD27 ${ }^{+} \mathrm{CD} 38^{\text {high }}$ ) and memory (IgD-IgM-CD27 ${ }^{+} \mathrm{CD} 38^{\text {low }}$ ) B cell subpopulations. Single plasmablast B cells were sorted into 96-well PCR plates (Axygen) containing $5 \mu \mathrm{l} /$ well of ice-cold PBS with RNAse Inhibitor (Takara) by a MoFlo Astrios EQ (Beckman Coulter). The plates were sealed and stored at $-80^{\circ} \mathrm{C}$ until subjected to reverse transcription (SuperScript III First Strand Synthesis System, Invitrogen) and PCR to amplify antibody heavy and light variable genes. For memory B cells, approximately 25-50 cells were sorted into 96-well culture plates (Corning) containing $50 \mu 1$ culture medium (ClonaCell-HY Medium A, STEMCELL Technologies) supplemented with $2.5 \mu \mathrm{g} / \mathrm{ml}$ of CpG (ODN 2006, Invitrogen), $10 \mu \mathrm{g} / \mathrm{ml}$ of Chk2 inhibitor (Sigma-Aldrich), $10 \mathrm{ng} / \mathrm{ml}$ of recombinant IL21 (rIL21) (Sino Biological Inc.), irradiated heterologous human PBMCs, and clarified culture supernatants of B95.8 cells (ATCC) containing the infectious Epstein-Barr virus. Approximately 7-10 days later, cell culture supernatants were screened for binding to ZIKV E glycoprotein and/or neutralizing activities against ZIKV strain, GZ01. The positive culture was subjected to cellular RNA extraction, reverse transcription (SuperScript III First Strand Synthesis System, Invitrogen), and PCR to amplify antibody heavy and light variable genes.

For both single plasmablasts and memory B cells, the nested PCR reactions were performed using mixtures of primers designed to amplify all antibody heavy and light chain variable regions (53). The generated PCR products were purified using QiaexII gel extraction kit (Qiagen) and cloned into the backbone of antibody expression vectors containing the constant regions of human $\operatorname{IgG1}$ (53). The paired antibody expression plasmids were transiently transfected into 293T cells, and supernatants were screened for binding to ZIKV E glycoprotein and neutralizing activity against ZIKV GZ01. The positive clones were then sequenced for the variable regions of antibody heavy chain genes (VH) and light chain genes (VL) and analyzed using the IMGT/V-Quest program. Manufacture of the whole recombinant human IgG1 antibodies were conducted in $293 \mathrm{~T}$ cells (ATCC) by transient transfection and purification by affinity chromatography using Protein A agarose. The concentration was determined by BCA Protein Assay Kit (Thermo Fisher Scientific). The expression clones encoding the control antibodies previously isolated by others from ZIKV- (ZV-48 and ZV-67) or DENV-infected (C8) subjects were synthesized and confirmed by sequencing before production in 293T cells and purified by protein A chromatograph. Control mAb 4G2 recognizing flavivirus group antigen was provided by Jing An from Capital Medical University, Beijing, China. MAb MERS-4 previously isolated by us (54) against MERS-CoV was used as a negative control.

Plasma profiling and epitope mapping by domain-specific E glycoprotein expressed on the yeast surface. Yeast clones expressing the domain-specific E glycoprotein were constructed as previously reported (38). In brief, the gene sequences encoding the DI/II (1-301aa) or DIII (302-404aa) of ZIKV, DENV1, and DENV2 were cloned into the yeast surface display vector pCTCON2. The rationale behind such design 
lies in the previous studies where DI/II and DIII were found to be structurally independent but connected through a poorly ordered and moderately conserved 11-amino acid linker (298-308aa). The breaking point between DI/II and DIII was located within the 11 residues between lysine $(\mathrm{K})$ at position 301 and glycine (G) at position 302 (Supplemental Figure 1), which are 100\% conservative among all of the known human flaviviruses such as ZIKV, DENV1-4, WNV, JEV, YFV, and TBEV (17, 31). The recombinant clones were then transformed into the $E$. coli-competent cells, amplified, extracted, and then further transformed into the competent yeast cell line EBY100 using electroporation. For surface expression of recombinant DI/II and DIII, EBY100 yeasts were first grown in SDCAA media at $30^{\circ} \mathrm{C}$ for 48 hours. At the exponential growth phase, yeasts were transferred to SGCAA media for induction of antigen expression at $20^{\circ} \mathrm{C}$ for 48 hours before incubating with either plasma samples or mAbs for subsequent analysis. Specifically, induced yeast cells $\left(1 \times 10^{6}\right.$ to $\left.1 \times 10^{7}\right)$ were collected by centrifugation $(3,500 \mathrm{~g}, 1$ minute $)$, washed twice with cold PBS, and incubated with either mAbs $(10 \mu \mathrm{g} / \mathrm{ml})$ or patient plasma $(1: 100$ dilution) on ice for 1 hour with occasional agitation. After being washed 3 times with cold PBS, the cells were incubated with PE-labeled anti-human IgG secondary antibody (1:200 dilution, rabbit anti-human IgG-PE, Santa Cruz Biotechnology Inc., sc3756) on ice for an additional 1 hour, washed again with PBS 3 times, and analyzed with FACS Calibur (BD Biosciences). C-Myc was a protein tag at the C-terminus of recombinant DI/II and DIII and was used for monitoring the expression under the induction conditions through anti-c-Myc chicken IgY (1:200 dilution, Thermo Fisher Scientific, A21281) and antichicken IgY FITC (1:200 dilution, Thermo Fisher Scientific, A11039)

Antibody neutralization and antibody-dependent enhancement assays. All ZIKV (GZ01; SZ-WIV01), DENV1 (Hawaii strain), and DENV2 (New Guinea strain; 16681 strain) viruses were grown in C6/36 Aedes albopictus cells and titrated on Vero cells before use. ZIKV GZ01 was isolated from Pt1 (35), and SZ-WIV01 was isolated from another imported case to China in 2016 by Wuhan Institute of Virology (Chinese Academy of Sciences), both of which are closely related with the strains circulating in the Americas and belong to the Asian lineage, including those strains identified from French Polynesia in 2013 (35). For neutralization assay, serial dilutions of plasma samples or mAbs were mixed with ZIKV (GZ01), DENV1 (Hawaii strain), or DENV2 (New Guinea strain) at $4^{\circ} \mathrm{C}$ for 1 hour before being applied to Vero cells in the 6-well culture plates. After 1-2 hour infection, antibody-virus mixture was aspirated and Vero cells were washed with PBS and overlayed with DMEM containing 2\% heat-inactivated FBS and 1\% SeaPlaque Agarose (Lonza, 50501). After 4-6 days, plaques were stained by $1 \%$ crystal violet and counted manually. For antibody-dependent enhancement assay, serially diluted mAbs were incubated with viruses ZIKV (SZ-WIV01) or DENV2 (16681) at $37^{\circ} \mathrm{C}$ for 1 hour before being added to K562 cells. Infected cells were then washed once, resuspended in RPMI 1640 medium supplemented with $2 \% \mathrm{FBS}$, and cultured at $37^{\circ} \mathrm{C}$ for 48 hours. Cells were harvested and intracellularly stained with a pan-flavivirus antibody 4G2 (provided by Jing An, Capital Medical University) or the DENV E-specific antibody D1-11 (Santa Cruz Biotechnology, sc-65659) at $4^{\circ} \mathrm{C}$ for 30 minutes, followed by incubation with anti-mouse IgG Alexa Fluor 488 (Invitrogen, A11029) for another 30 minutes at $4^{\circ} \mathrm{C}$. The infection rate of cells was determined by flow cytometry. Fold enhancement was analyzed by comparison with the percentage of infected cells in the presence or absence of antibodies.

Plasma absorption experiments. To evaluate the contribution of domain-specific antibodies to overall neutralization activity, plasma samples were incubated with yeast clones expressing ZIKV envelope DI/II or DIII to specifically absorb and remove antibodies targeting these regions. Induced yeast clones were centrifuged before being added to 1:50 diluted plasma with 10\% FBS DMEM. The mixture was incubated on a rotary shaker at $4^{\circ} \mathrm{C}$ overnight. The same procedure was repeated several times by adding fresh yeast during each cycle of absorption until no antibody response was detectable against the corresponding yeast measured by FACS. The resultant plasma samples were adjusted to neutral with PBS, filtered (0.22 mm diameter filter; Corning), and then evaluated for their neutralizing activity. The percent of contribution was calculated by comparing the neutralizing activity with plasma samples that were treated with irrelevant CD20 yeast.

Epitope mapping of $m A$ bs by SPR. For epitope mapping, His Capture Kit was used according to manufacture's instructions (GE 28-9950-56). Anti-histidine antibodies were immobilized to CM5 sensor chip via Amine group. Approximately $10 \mu \mathrm{g} / \mathrm{ml}$ ZIKV E protein were injected as ligand, and then 2 different $\mathrm{mAbs}$ were injected sequentially to monitor for additional binding activity to determine whether the $2 \mathrm{mAbs}$ recognized the separate or closely situated epitopes. 
Quantitative measurement of viral genome by TaqMan quantitative PCR. Total RNA was isolated from the whole blood of AG6 mouse using an RNeasy Mini Kit (74106, Qiagen) and reverse transcribed into cDNA using an iScript cDNA synthesis kit (170-8890, Bio-Rad). Viral genomes were quantified via TaqMan qPCR amplification of ZIKV genes. The primers and probes used for this analysis are shown as follows: ZIKV-F 5' - CCGCTGCCCAACACAAG - 3', ZIKV-R 5' - CCACTAACGTTCTTTTGCAGACAT - 3', and ZIKV-probe 5' - AGCCTACCTTGACAAGCARTCAGACACTCAA - 3' (5' FAM, 3' TAMRA).

Assessment of therapeutic activity of $m A$ bs in mice. C57BL/ 6 mice deficient in IFN $\alpha,-\beta$, and $-\gamma$ receptors (AG6 mouse) were purchased from Institute Pasteur of Shanghai, Chinese Academy of Sciences. The mice were bred and maintained under a specific pathogen-free animal facility at Tsinghua University. AG6 mice aged 4-6 weeks were challenged with $1 \times 10^{4}$ pfu ZIKV (GZ01 strain) via the i.p. route and followed for 14 days. mAbs (300 $\mu \mathrm{g}$; ZK2B10, ZK8-4, or control antibody MERS-4) diluted in PBS were administered via i.p. 1 day after virus challenge. Weights were recorded daily. On day 5 and day 13 after challenge, blood was collected from each animal for viral load measurement.

Statistics. In ELISA analysis of plasma binding, half-maximal effective concentrations $\left(\mathrm{EC}_{50}\right)$ were calculated using the dose-response stimulation model in GraphPad Prism (GraphPad Software Inc.). In virus neutralizing assay, $\mathrm{IC}_{50}$ for each $\mathrm{mAb}$ were calculated using the dose-response inhibition model in Graph$\mathrm{Pad}$ Prism. In mice challenge and protection experiments, statistical significance was analyzed by a oneway ANOVA with a Dunnett's multiple comparisons test $(* * *<0.001)$ via GraphPad Prism.

Study approval. The human study was approved by the Ethical Committee of the Guangzhou Eighth People's Hospital, Guangzhou Medical University. The research was conducted in strict accordance with the Chinese government rules and regulations for the protection of human subjects. The study subjects provided the written informed consents for research use of their blood samples. All procedures with animals were undertaken according to Experimental Animal Welfare and Ethics Committee of Tsinghua University.

\section{Author contributions}

LY, RW, FG, XJ, GC, FZ, and L. Zhang conceived of the research, designed the study, interpreted data, and wrote the manuscript. LY, RW, FG, ML, JL, L. Zhao, YW, HW, QZ, Y. Li, PZ, RZ, and Y. Liu performed experiments. JW, WH, CY, XT, YG, CFQ, LC, and XS managed patients, collected clinical samples, cultured and provided relevant viral strains for analysis, and wrote the relevant sections in the manuscript. All authors reviewed the results and approved the final version of the manuscript. LY and RW contributed equally to this research, and FZ and L. Zhang are co-senior authors in directing the entire research.

\section{Acknowledgments}

The project was supported by Guangzhou Science and Technology Program for Public Wellbeing (2014Y200185, 2014Y2-00550, and 201508020263), the Special Program of Guangdong Provincial Department of Science and Technology (2016A020248001), the Guangzhou Health Care and Cooperation Innovation Major grant, the National Natural Science Foundation Award (81530065 and 81590762), the National Key Plan for Scientific Research and Development of China (2016YFC1200902), and Ministry of Science and Technology of China (2014CB542500-03).

Address corresponding to: Linqi Zhang, Comprehensive AIDS Research Center, Department of Basic Medical Sciences, School of Medicine, Tsinghua University, Beijing, 100084, China. Phone: 86.10.62788131; Email: zhanglinqi@tsinghua.edu.cn. Or to: Fuchun Zhang, Guangzhou Eighth People's Hospital, Guangzhou Medical University, 627 Dongfeng Road East, Guangzhou 510060, China. Phone: 86-13570037328; Email: gz8hzfc@126.com.

1. Brasil P, et al. Guillain-Barré syndrome associated with Zika virus infection. Lancet. 2016;387(10026):1482.

2. Cao-Lormeau VM, et al. Guillain-Barré Syndrome outbreak associated with Zika virus infection in French Polynesia: a case-control study. Lancet. 2016;387 (10027):1531-1539.

3. Kleber de Oliveira W, et al. Increase in Reported Prevalence of Microcephaly in Infants Born to Women Living in Areas with Confirmed Zika Virus Transmission During the First Trimester of Pregnancy - Brazil, 2015. MMWR Morb Mortal Wkly Rep. 2016;65 (9):242-247.

4. Rasmussen SA, Jamieson DJ, Honein MA, Petersen LR. Zika Virus and Birth Defects--Reviewing the Evidence for Causality. N Engl J Med. 2016;374(20):1981-1987.

5. Mlakar J, et al. Zika Virus Associated with Microcephaly. N Engl J Med. 2016;374(10):951-958. 
6. Oehler E, et al. Zika virus infection complicated by Guillain-Barre syndrome--case report, French Polynesia, December 2013. Euro Surveill. 2014;19 (9):20720.

7. Dick GW, Kitchen SF, Haddow AJ. Zika virus. I. Isolations and serological specificity. Trans R Soc Trop Med Hyg. 1952;46(5):509-520

8. Faria NR, et al. Zika virus in the Americas: Early epidemiological and genetic findings. Science. 2016;352 (6283):345-349.

9. Wikan N, Smith DR. Zika virus: history of a newly emerging arbovirus. Lancet Infect Dis. 2016;16(7):e119-e126.

10. Singer M. The spread of Zika and the potential for global arbovirus syndemics. Glob Public Health. 2017;12 (1):1-18.

11. Voss A, Kluytmans J, Pittet D. A new journal and new global perspective on infection control and public health. Antimicrob Resist Infect Control. 2012;1 (1):4.

12. Corti D, Lanzavecchia A. Broadly neutralizing antiviral antibodies. Annu Rev Immunol. 2013;31:705-742.

13. Burton DR, Hangartner L. Broadly Neutralizing Antibodies to HIV and Their Role in Vaccine Design. Annu Rev Immunol. 2016;34:635-659.

14. Pierson TC, et al. The stoichiometry of antibody-mediated neutralization and enhancement of West Nile virus infection. Cell Host Microbe. 2007;1 (2):135-145.

15. Beltramello M, et al. The human immune response to Dengue virus is dominated by highly cross-reactive antibodies endowed with neutralizing and enhancing activity. Cell Host Microbe. 2010;8 (3):271-283.

16. Barba-Spaeth G, et al. Structural basis of potent Zika-dengue virus antibody cross-neutralization. Nature. 2016;536(7614):48-53.

17. Zhao H, et al. Structural Basis of Zika Virus-Specific Antibody Protection. Cell. 2016;166(4):1016-1027.

18. Wahala WM, Silva AM. The human antibody response to dengue virus infection. Viruses. 2011;3(12):2374-2395.

19. Dejnirattisai W, et al. A new class of highly potent, broadly neutralizing antibodies isolated from viremic patients infected with dengue virus. Nat Immunol. 2015;16(2):170-177.

20. Fibriansah G, Lok SM. The development of therapeutic antibodies against dengue virus. Antiviral Res. 2016;128:7-19.

21. Sirohi D, et al. The $3.8 \AA$ A resolution cryo-EM structure of Zika virus. Science. 2016;352 (6284):467-470.

22. Kostyuchenko VA, et al. Structure of the thermally stable Zika virus. Nature. 2016;533 (7603):425-428.

23. Zhang X, et al. Cryo-EM structure of the mature dengue virus at 3.5-Å resolution. Nat Struct Mol Biol. 2013;20(1):105-110.

24. Pierson TC, Diamond MS. Degrees of maturity: the complex structure and biology of flaviviruses. Curr Opin Virol. 2012;2 (2):168-175.

25. Zhang Y, et al. Conformational changes of the flavivirus E glycoprotein. Structure. 2004;12 (9):1607-1618.

26. Mukhopadhyay S, Kim BS, Chipman PR, Rossmann MG, Kuhn RJ. Structure of West Nile virus. Science. 2003;302(5643):248

27. Kuhn RJ, et al. Structure of dengue virus: implications for flavivirus organization, maturation, and fusion. Cell. $2002 ; 108$ (5):717-725.

28. Dai L, et al. Structures of the Zika Virus Envelope Protein and Its Complex with a Flavivirus Broadly Protective Antibody. Cell Host Microbe. 2016;19 (5):696-704.

29. Kaufmann B, et al. Neutralization of West Nile virus by cross-linking of its surface proteins with Fab fragments of the human monoclonal antibody CR4354. Proc Natl Acad Sci USA. 2010;107 (44):18950-18955.

30. Sapparapu G, et al. Neutralizing human antibodies prevent Zika virus replication fetal disease in mice. Nature. 2016;540(7633):443-447.

31. Stettler K, et al. Specificity, cross-reactivity, and function of antibodies elicited by Zika virus infection. Science. 2016;353 (6301):823-826.

32. Wang Q, et al. Molecular determinants of human neutralizing antibodies isolated from a patient infected with Zika virus. Sci Transl Med. 2016;8 (369):369ra179.

33. Rouvinski A, et al. Recognition determinants of broadly neutralizing human antibodies against dengue viruses. Nature. 2015;520 (7545):109-113.

34. Austin SK, Dowd KA. B cell response and mechanisms of antibody protection to West Nile virus. Viruses. 2014;6(3):1015-1036

35. Zhang FC, Li XF, Deng YQ, Tong YG, Qin CF. Excretion of infectious Zika virus in urine. Lancet Infect Dis. 2016;16(6):641-642.

36. Sukupolvi-Petty S, et al. Type- and subcomplex-specific neutralizing antibodies against domain III of dengue virus type 2 envelope protein recognize adjacent epitopes. J Virol. 2007;81 (23):12816-12826.

37. Zuo T, et al. Comprehensive analysis of antibody recognition in convalescent humans from highly pathogenic avian influenza H5N1 infection. Nat Commun. 2015;6:8855.

38. Zuo T, et al. Comprehensive analysis of pathogen-specific antibody response in vivo based on an antigen library displayed on surface of yeast. J Biol Chem. 2011;286 (38):33511-33519.

39. Smith SA, et al. The potent and broadly neutralizing human dengue virus-specific monoclonal antibody $1 \mathrm{C} 19$ reveals a unique cross-reactive epitope on the bc loop of domain II of the envelope protein. MBio. 2013;4 (6):e00873-e00813.

40. Liu J, et al. Flavivirus NS1 protein in infected host sera enhances viral acquisition by mosquitoes. Nat Microbiol. 2016;1 (9): 16087.

41. Orozco S, et al. Characterization of a model of lethal dengue virus 2 infection in C57BL/6 mice deficient in the alpha/beta interferon receptor. J Gen Virol. 2012;93 (Pt 10):2152-2157.

42. Sabin AB. Research on dengue during World War II. Am J Trop Med Hyg. 1952;1(1):30-50.

43. Priyamvada L, et al. Human antibody responses after dengue virus infection are highly cross-reactive to Zika virus. Proc Natl Acad Sci USA. 2016;113(28):7852-7857.

44. Imrie A, et al. Antibody to dengue 1 detected more than 60 years after infection. Viral Immunol. 2007;20 (4):672-675.

45. Dejnirattisai W, et al. Dengue virus sero-cross-reactivity drives antibody-dependent enhancement of infection with zika virus. Nat Immunol. 2016;17 (9):1102-1108.

46. Swanstrom JA, et al. Dengue Virus Envelope Dimer Epitope Monoclonal Antibodies Isolated from Dengue Patients Are Protective against Zika Virus. MBio. 2016;7(4):e01123.

47. Chan KR, et al. Ligation of Fc gamma receptor IIB inhibits antibody-dependent enhancement of dengue virus infection. Proc Natl Acad Sci USA. 2011;108 (30):12479-12484.

48. Kam YW, et al. Cross-reactive dengue human monoclonal antibody prevents severe pathologies and death from Zika virus 
infections. JCI Insight. 2017;2(8):e92428.

49. Dejnirattisai W, et al. Cross-reacting antibodies enhance dengue virus infection in humans. Science. 2010;328 (5979):745-748.

50. Bardina SV, et al. Enhancement of Zika virus pathogenesis by preexisting antiflavivirus immunity. Science. 2017;356(6334):175-180.

51. Zhang Y, et al. Highly diversified Zika viruses imported to China, 2016. Protein Cell. 2016;7 (6):461-464.

52. Guan Y, et al. Discordant memory B cell and circulating anti-Env antibody responses in HIV-1 infection. Proc Natl Acad Sci USA. 2009;106(10):3952-3957.

53. Tiller T, Meffre E, Yurasov S, Tsuiji M, Nussenzweig MC, Wardemann H. Efficient generation of monoclonal antibodies from single human B cells by single cell RT-PCR and expression vector cloning. J Immunol Methods. 2008;329 (1-2):112-124.

54. Jiang $\mathrm{L}$, et al. Potent neutralization of MERS-CoV by human neutralizing monoclonal antibodies to the viral spike glycoprotein. Sci Transl Med. 2014;6 (234):234ra59. 